\title{
OSCILLATION OF ONE ORDER NEUTRAL DIFFERENTIAL EQUATION WITH IMPULSES
}

\author{
Jinfa Cheng And Yuming Chu
}

ABSTRACT. Explicit sufficient conditions are established for the oscillation of the one order neutral differential equations with impulsive

$$
\begin{gathered}
\left(x(t)+\sum_{i=1}^{n} c_{i} x\left(t-\sigma_{i}\right)\right)^{\prime}+p x(t-\tau)=0, t \neq t_{k}, \\
\Delta\left(x\left(t_{k}\right)+\sum_{i=1}^{n} c_{i} x\left(t_{k}-\sigma_{i}\right)\right)+p_{0} x\left(t_{k}-\tau\right)=0,
\end{gathered}
$$

where $c_{i} \geq 0, i=1,2, \ldots, n, p \tau>0, p_{0} \tau>0, \Delta\left(x_{k}\right)=x\left(t_{k}^{+}\right)-x\left(t_{k}\right)$. Explicit sufficient and necessary condition are established when $c_{i}=0, i=$ $1,2, \ldots, n$.

\section{Introduction}

The oscillation theory of impulsive differential equations is one of the directions which initiated the investigations on the qualitive properties of the differential equations. It was published in 1989 the paper of K. Gopalsamy and B. G. Zhang [7] where the first investigation on the oscillatory properties of impulsive differential equation was carried out. Unfortunately, this work was not followed by another publications.

During the last several years, D. Bainov and D. P. Mishev have studied the oscillatory properties of various of impulsive differential equations. The monograph [5] is the first book to present systematically the result known up to 1998, and to demonstrate how well-know mathematical techniques and methods after suitable modification, can be applied in proving oscillatory theorems for impulsive differential equations. For oscillation theory of differential equation, we prefer to the monographs $[1,2,3,4,6,8]$.

Received June 26, 2007.

2010 Mathematics Subject Classification. 34A37.

Key words and phrases. oscillation, impulses, characteristic system.

The research is partly supported by the N. S. Foundation of China under grant 11071069 and the Innovation Team Foundation of the Department of Education of Zhejiang Province under grant T200924. 
Consider the periodic linear impulsive neutral differential equation with delay

$$
\begin{aligned}
\left(x(t)+\sum_{i=1}^{n} c_{i} x\left(t-\sigma_{i}\right)\right)^{\prime}+p x(t-\tau) & =0, \quad t \neq t_{k}, \\
\Delta\left(x\left(t_{k}\right)+\sum_{i=1}^{n} c_{i} x\left(t_{k}-\sigma_{i}\right)\right)+p_{0} x\left(t_{k}-\tau\right) & =0,
\end{aligned}
$$

where $c_{i} \geq 0, i=1,2, \ldots, n, \Delta x\left(t_{k}\right)=x\left(t_{k}^{+}\right)-x\left(t_{k}^{-}\right)$.

We assume the validity of the following conditions:

H1. $p>0,0<p_{0}<1, \tau>0$.

$H 2$. There exist $m, m_{i} \in \mathbb{N}$ such that

$$
i\left[t-\sigma_{i}, t\right)=m_{i}, i[t-\tau, t)=m, t \in \mathbb{R} .
$$

Here $i\langle a, b\rangle$ denotes the number of the points $t_{k}$, lying in the interval $\langle a, b\rangle$.

We are looking for a positive solution of the equation (1.1) in the form

$$
x(t)=e^{-\lambda t}(1-\mu)^{i[0, t]},
$$

where $\lambda \in \mathbb{R}, \mu<1$.

We substitute (1.2) into (1.1) and obtain that $x(t)$ has the form (1.2) if

$$
\begin{aligned}
\lambda+\sum_{i=1}^{n} c_{i} \lambda e^{\lambda \sigma_{i}}(1-\mu)^{-m_{i}} & =p e^{\lambda \tau}(1-\mu)^{-m} \\
\mu+\sum_{i=1}^{n} c_{i} \mu e^{\lambda \sigma_{i}}(1-\mu)^{-m_{i}} & =p_{0} e^{\lambda \tau}(1-\mu)^{-m} .
\end{aligned}
$$

The system (1.3) is called characteristic system corresponding to the equation (1.1).

It is easy to see that the solution $(\lambda, \mu)$ of Eq.(1.3) satisfies $\mu=\frac{p_{0}}{p} \lambda$. Moreover the system (1.3) has a solution $(\lambda, \mu)$ with $\mu<1$ if and only if the characteristic equation

$$
H(\lambda)=-\lambda-\sum_{i=1}^{n} c_{i} \lambda e^{\lambda \sigma_{i}}\left(1-\frac{p_{0}}{p} \lambda\right)^{-m_{i}}+p e^{\lambda t}\left(1-\frac{p_{0}}{p} \lambda\right)^{-m}=0
$$

has a solution $\lambda \in\left(0, \frac{p_{0}}{p}\right)$. The following basic result was established by $\mathrm{D}$. Bainov and P. Simeonov [5].

Theorem A. Let the conditions $\mathrm{H} 1$ and $\mathrm{H} 2$ be fulfilled. Then the following assertions are equivalent:

(i) The equation (1.4) has no solution $\lambda \in\left(0, \frac{p_{0}}{p}\right)$.

(ii) The characteristic system (1.3) has no solution $(\lambda, \mu)$ with $\mu<1$.

(iii) Each regular solution of the equation (1.1) is oscillatory. 
Similarly, we consider the periodic linear impulsive neutral differential equation with advance

$$
\begin{aligned}
\left(x(t)+\sum_{i=1}^{n} c_{i} x\left(t+\sigma_{i}\right)\right)^{\prime}+p x(t+\tau) & =0, \quad t \neq t_{k}, \\
\Delta\left(x\left(t_{k}\right)+\sum_{i=1}^{n} c_{i} x\left(t_{k}+\sigma_{i}\right)\right)+p_{0} x\left(t_{k}+\tau\right) & =0 .
\end{aligned}
$$

We introduce the following conditions:

H3. $p<0, p_{0}<0, \tau>0$.

H4. There exist $n, n_{1} \in \mathbb{N}$ such that

$$
i\left[t, t+\sigma_{i}\right]=n_{i}, i[t, t+\tau]=n, t \in \mathbb{R} .
$$

We look for a positive solution of the equation (1.5) with form (1.2).

Substituting (1.2) into (1.5) we obtain that $x(t)$ is a solution of Eq.(1.5) if

$$
\begin{aligned}
\lambda+\sum_{i=1}^{n} c_{i} \lambda e^{-\lambda \sigma_{i}}(1-\mu)^{n_{i}} & =p e^{-\lambda \tau}(1-\mu)^{n}, \\
\mu+\sum_{i=1}^{n} c_{i} \mu e^{-\lambda \sigma_{i}}(1-\mu)^{n_{i}} & =p_{0} e^{-\lambda \tau}(1-\mu)^{n} .
\end{aligned}
$$

The system (1.6) is called characteristic system corresponding to the equation (1.5).

We get the solution $(\lambda, \mu)$ of system (1.6) $\mu=\frac{p_{0}}{p} \lambda$, and the system (1.6) has a solution $(\lambda, \mu)$ with $\mu<1$ if and only if the characteristic equation

$$
H(\lambda)=-\lambda-\sum_{i=1}^{n} c_{i} \lambda e^{-\lambda \sigma_{i}}\left(1-\frac{p_{0}}{p} \lambda\right)^{n_{i}}+p e^{-\lambda \tau}\left(1-\frac{p_{0}}{p} \lambda\right)^{n}=0
$$

has a solution $\lambda$ for which $\lambda<\frac{p}{p_{0}}$.

Theorem B. Let the conditions $H 3$ and $H 4$ be fulfilled. Then the following assertions are equivalent:

(j) The equation (1.7) has no solution $\lambda<0$.

(jj) The characteristic system (1.6) has no solution $(\lambda, \mu)$ with $\mu<0$.

(jjj) Each regular solution of the equation (1.5) is oscillatory.

Just like on the case when we investigate one order linear differential equations without impulses, it is very significant to obtain explicit sufficient conditions or necessary and sufficient conditions for the oscillation, see the monograph [8]. Although Theorem A and Theorem B are fundamental results, they are not easy to apply. Therefore, in this paper, we give explicit sufficient conditions for oscillation of Eq.(1.1) and Eq.(1.5), necessary and sufficient conditions for oscillation of Eq.(1.1) and Eq.(1.5) when $c_{i}=0, i=1,2, \ldots, n$. It is notable that when Eq.(1.1) and Eq.(1.5) are reduced to differential equations without 
impulses, our results improve or coincide with the corresponding theorems in $[8]$.

\section{Main results and proofs}

Theorem 1. Let the conditions $H 1$ and $H 2$ be fulfilled. Then every solution of Eq.(1.1) oscillates if

$$
-\lambda_{0}\left(1-\lambda_{0}\right)^{m} e^{-\frac{p}{p_{0}} \lambda_{0} \tau}-\sum_{i=1}^{n} c_{i} \lambda_{i}\left(1-\lambda_{i}\right)^{m-m_{i}} e^{-\frac{p}{p_{0}} \lambda_{i}\left(\tau-\sigma_{i}\right)}+p_{0}>0,
$$

where

$$
\begin{gathered}
\lambda_{0}=\frac{\frac{p \tau}{p_{0}}+m+1-\sqrt{\left(\frac{p \tau}{p_{0}}+m+1\right)^{2}-\frac{4 p \tau}{p_{0}}}}{\frac{\frac{2 p \tau}{p_{0}}}{\lambda_{0}}}, \\
\lambda_{i}=\frac{\frac{p\left(\tau-\sigma_{i}\right)}{p_{0}}+m-m_{i}+1-\sqrt{\left(\frac{p\left(\tau-\sigma_{i}\right)}{p_{0}}+m-m_{i}+1\right)^{2}-\frac{4 p\left(\tau-\sigma_{i}\right)}{p_{0}}}}{\frac{2 p\left(\tau-\sigma_{i}\right)}{p_{0}}} .
\end{gathered}
$$

Proof. By Theorem A, for equation

$$
H(\lambda)=-\lambda-\sum_{i=1}^{n} c_{i} \lambda e^{\lambda \sigma_{i}}\left(1-\frac{p_{0}}{p} \lambda\right)^{-m_{i}}+p e^{\lambda \tau}\left(1-\frac{p_{0}}{p} \lambda\right)^{-m}=0,
$$

if it has no solution $\lambda \in\left(0, \frac{p_{0}}{p}\right)$, then each solution is oscillatory. From $H(\lambda)=$ 0 , we have

$$
-\frac{p_{0}}{p} \lambda\left(1-\frac{p_{0}}{p} \lambda\right)^{m}-\sum_{i=1}^{n} c_{i} \frac{p_{0}}{p} \lambda e^{\lambda \sigma_{i}}\left(1-\frac{p_{0}}{p} \lambda\right)^{m-m_{i}}+p_{0} e^{\lambda \tau}=0 .
$$

Let $\frac{p_{0}}{p} \lambda=\tilde{\lambda}$, then $\tilde{\lambda} \in(0,1)$. For the sake of convenience, we still denote $\tilde{\lambda}$ by $\lambda$. Then Eq.(2.2) becomes

$$
-\lambda(1-\lambda)^{m}-\sum_{i=1}^{n} c_{i} \lambda e^{\frac{p}{p_{0}} \lambda \sigma_{i}}(1-\lambda)^{m-m_{i}}+p_{0} e^{\frac{p}{p_{0}} \lambda \tau}=0 .
$$

Set

$$
F(\lambda)=-\lambda(1-\lambda)^{m} e^{-\frac{p}{p_{0}} \lambda \tau}-\sum_{i=1}^{n} c_{i} \lambda(1-\lambda)^{m-m_{i}} e^{-\frac{p}{p_{0}} \lambda\left(\tau-\sigma_{i}\right)}+p_{0} .
$$

If $F(\lambda)=0$ has no solution in $(0,1)$, then from Theorem A we know that each solution is oscillatory. Let

$$
\begin{gathered}
G(\lambda)=-\lambda(1-\lambda)^{m} e^{-\frac{p}{p_{0}} \lambda \tau}, \\
G_{i}(\lambda)=-c_{i} \lambda(1-\lambda)^{m-m_{i}} e^{-\frac{p}{p_{0}} \lambda\left(\tau-\sigma_{i}\right)} .
\end{gathered}
$$

Then

$$
G^{\prime}(\lambda)=(1-\lambda)^{m-1} e^{-\frac{p}{p_{0}} \lambda \tau}\left[-\frac{p \tau}{p_{0}} \lambda^{2}+\left(\frac{p \tau}{p_{0}}+m+1\right) \lambda-1\right] .
$$


Denote

$$
\begin{aligned}
I(\lambda) & =-\left[\frac{p \tau}{p_{0}} \lambda^{2}-\left(\frac{p \tau}{p_{0}}+m+1\right) \lambda+1\right], \\
\Delta & =\left(\frac{p \tau}{p_{0}}+m+1\right)^{2}-4 \frac{p \tau}{p_{0}} .
\end{aligned}
$$

Obviously, $\Delta>\left(\frac{p \tau}{p_{0}}+m-1\right)^{2}$. If $m \neq 0$, then $\Delta>0$ and equation $I(\lambda)=0$ have two different real roots:

It is easy to verify that

$$
\begin{aligned}
& \lambda_{0}=\frac{\frac{p \tau}{p_{0}}+m+1-\sqrt{\Delta}}{2 \frac{p \tau}{p_{0}}}, \\
& \tilde{\lambda}_{0}=\frac{\frac{p \tau}{p_{0}}+m+1+\sqrt{\Delta}}{2 \frac{p \tau}{p_{0}}} .
\end{aligned}
$$

$$
0<\lambda_{0}<1<\tilde{\lambda}_{0}
$$

We can delete $\tilde{\lambda}_{0}$ since $\tilde{\lambda}_{0}>1$. Therefore we obtain the following conclusions:

1. If $0<\lambda<\lambda_{0}$, then $I(\lambda)<0$, that is $G^{\prime}(\lambda)<0$ and $G(\lambda)$ is decreasing;

2. If $\lambda_{0}<\lambda<1$, then $I(\lambda)<0$, that is $G^{\prime}(\lambda)>0$ and $G(\lambda)$ is increasing. Thus $G(\lambda)$ attain its minimum value at $\lambda=\lambda_{0}$ and

$$
G(\lambda)=-\lambda(1-\lambda)^{m} e^{-\frac{p}{p_{0}} \lambda \tau} \geq-\lambda_{0}\left(1-\lambda_{0}\right)^{m} e^{-\frac{p}{p_{0}} \lambda_{0} \tau} .
$$

Similarly, we conclude that $G_{i}(\lambda)$ attain its minimum value at $\lambda=\lambda_{i}$ and

$$
G_{i}(\lambda)=-c_{i} \lambda(1-\lambda)^{m-m_{i}} e^{-\frac{p}{p_{0}} \lambda\left(\tau-\sigma_{i}\right)} \geq-c_{i} \lambda_{i}\left(1-\lambda_{i}\right)^{m-m_{i}} e^{-\frac{p}{p_{0}} \lambda_{i}\left(\tau-\sigma_{i}\right)} .
$$

In view of the condition $(2.1)$, we get $F(\lambda)>0$ for all $\lambda \in(0,1)$, so there does not exists real root in $(0,1)$ for equation $F(\lambda)=0$ and Eq.(1.1) is oscillatory.

Remark 1. If $m=0, m_{i}=0, i=1,2, \ldots, n$, then Eq.(1.1) is a differential equation without impulses

$$
\left(x(t)+\sum_{i=1}^{n} c_{i} x\left(t-\sigma_{i}\right)\right)^{\prime}+p x(t-\tau)=0,
$$

and $\lambda_{0}=\frac{p_{0}}{p \tau}, \lambda_{i}=\frac{p_{0}}{p\left(\tau-\sigma_{i}\right)}$. The condition (2.1) becomes

$$
-\frac{p_{0}}{p \tau} e^{-1}-\sum_{i=1}^{n} c_{i} \frac{p_{0}}{p\left(\tau-\sigma_{i}\right)} e^{-1}+p_{0}>0 .
$$

That is

$$
\frac{1}{\tau}+\sum_{i=1}^{n} c_{i} \frac{1}{\tau-\sigma_{i}}<p e .
$$

Further, if $c_{2}=c_{3}=\cdots=c_{n}=0$, then

$$
\frac{1}{\tau}+c_{1} \frac{1}{\tau-\sigma_{1}}<p e,
$$

which is a improvement of Theorem 6.1.3 in [8]. 
Theorem 2. Let the conditions $\mathrm{H}_{3}$ and $\mathrm{H}_{4}$ be fulfilled. Then every solution of Eq.(1.5) oscillates if

$$
\lambda_{0}\left(1+\lambda_{0}\right)^{-n} e^{-\frac{p}{p_{0}} \lambda \tau}+\sum_{i=1}^{n} c_{i} \lambda_{i}\left(1+\lambda_{i}\right)^{-\left(n-n_{i}\right)} e^{-\frac{p}{p_{0}} \lambda_{i}\left(\tau-\sigma_{i}\right)}+p_{0}<0
$$

where

$$
\begin{gathered}
\lambda_{0}=\frac{-\left(\frac{p \tau}{p_{0}}+n-1\right)+\sqrt{\left(\frac{p \tau}{p_{0}}+n-1\right)^{2}+\frac{4 p \tau}{p_{0}}}}{\frac{2 p \tau}{p_{0}}}, \\
\lambda_{i}=\frac{-\left(\frac{p\left(\tau-\sigma_{i}\right)}{p_{0}}+n-n_{i}-1\right)+\sqrt{\left(\frac{p\left(\tau-\sigma_{i}\right)}{p_{0}}+n-n_{i}-1\right)^{2}+\frac{4 p \tau}{p_{0}}}}{\frac{2 p\left(\tau-\sigma_{i}\right)}{p_{0}}} .
\end{gathered}
$$

Proof. By Theorem B, if

$$
H(\lambda)=-\lambda-\sum_{i=1}^{n} c_{i} \lambda e^{-\lambda \sigma_{i}}\left(1-\frac{p_{0}}{p} \lambda\right)^{n_{i}}+p e^{-\lambda \tau}\left(1-\frac{p_{0}}{p} \lambda\right)^{n}=0
$$

has no solution $\lambda<0$, then each regular solution of Eq.(1.5) oscillates. It is equivalent to the equation

$$
H(\lambda)=\lambda+\sum_{i=1}^{n} c_{i} \lambda e^{\lambda \sigma_{i}}\left(1+\frac{p_{0}}{p} \lambda\right)^{n_{i}}+p e^{\lambda \tau}\left(1+\frac{p_{0}}{p} \lambda\right)^{n}=0
$$

has no real root $\lambda \in(0, \infty)$. We have

$$
H(\infty)<0, H(0)=p<0 .
$$

Equation $H(\lambda)=0$ is equivalent to

$$
\frac{p_{0}}{p} \lambda+\sum_{i=1}^{n} c_{i} \frac{p_{0}}{p} \lambda e^{\lambda \sigma_{i}}\left(1+\frac{p_{0}}{p} \lambda\right)^{n_{i}}+p_{0} e^{\lambda \tau}\left(1+\frac{p_{0}}{p} \lambda\right)^{n}=0 .
$$

Let $\frac{p_{0}}{p} \lambda=\tilde{\lambda}, \lambda=\frac{p}{p_{0}} \tilde{\lambda}$. Then Eq.(2.5) becomes

$$
\tilde{\lambda}+\sum_{i=1}^{n} c_{i} \tilde{\lambda} e^{\frac{p}{p_{0}} \tilde{\lambda} \sigma_{i}}(1+\tilde{\lambda})^{n_{i}}+p_{0} e^{\frac{p}{p_{0}} \tilde{\lambda} \tau}(1+\tilde{\lambda})^{n}=0 .
$$

For the sake of convenience, we still denote $\tilde{\lambda}$ by $\lambda, \lambda \in(0, \infty)$. Then

$$
F(\lambda)=\lambda(1+\lambda)^{-n} e^{-\frac{p}{p_{0}} \lambda \tau}+\sum_{i=1}^{n} c_{i} \lambda(1+\lambda)^{-\left(n-n_{i}\right)} e^{-\frac{p}{p_{0}} \lambda\left(\tau-\sigma_{i}\right)}+p_{0}
$$

Let

$$
\begin{gathered}
G(\lambda)=\lambda(1+\lambda)^{-n} e^{-\frac{p}{p_{0}} \lambda \tau}, \\
G_{i}(\lambda)=c_{i} \lambda(1+\lambda)^{-\left(n-n_{i}\right)} e^{-\frac{p}{p_{0}} \lambda\left(\tau-\sigma_{i}\right)} .
\end{gathered}
$$

Then

$$
G^{\prime}(\lambda)=e^{-\frac{p}{p_{0}} \lambda \tau}(1+\lambda)^{-n-1}\left[-\frac{p \tau}{p_{0}} \lambda^{2}-\left(\frac{p \tau}{p_{0}}+n-1\right) \lambda+1\right] .
$$


Denote

$$
\begin{aligned}
I(\lambda) & =\left[-\frac{p \tau}{p_{0}} \lambda^{2}-\left(\frac{p \tau}{p_{0}}+n-1\right) \lambda+1\right], \\
\Delta & =\left(\frac{p \tau}{p_{0}}+n-1\right)^{2}+\frac{4 p \tau}{p_{0}}>0 .
\end{aligned}
$$

Then $I(\lambda)$ have two different roots:

$$
\begin{aligned}
& \lambda_{0}=\frac{-\left(\frac{p \tau}{p_{0}}+n-1\right)+\sqrt{\left(\frac{p \tau}{p_{0}}+n-1\right)^{2}+\frac{4 p \tau}{p_{0}}}}{\frac{2 p \tau}{p_{0}}}>0, \\
& \tilde{\lambda}_{0}=\frac{-\left(\frac{p \tau}{p_{0}}+n-1\right)-\sqrt{\left(\frac{p \tau}{p_{0}}+n-1\right)^{2}+\frac{4 p \tau}{p_{0}}}}{\frac{2 p \tau}{p_{0}}}<0 .
\end{aligned}
$$

We delete $\tilde{\lambda}_{0}$ since $\tilde{\lambda}_{0}<0$. So we have shown the validity of the following conclusions:

1. If $0<\lambda<\lambda_{0}$, then $I(\lambda)>0$, that is $G^{\prime}(\lambda)>0$ and $G(\lambda)$ is increasing;

2. If $\lambda_{0}<\lambda<\infty$, then $I(\lambda)<0$, that is $G^{\prime}(\lambda)<0$ and $G(\lambda)$ is decreasing. Therefore $G(\lambda)$ attain its maximum value at $\lambda=\lambda_{0}$ and

$$
G(\lambda)=\lambda(1+\lambda)^{-n} e^{-\frac{p}{p_{0}} \lambda \tau} \leq \lambda_{1}\left(1+\lambda_{0}\right)^{-n} e^{-\frac{p}{p_{0}} \lambda_{0} \tau} .
$$

Similarly, we know that $G_{i}(\lambda)$ attain its maximum value at $\lambda=\lambda_{i}$ and

$$
G_{i}(\lambda)=c_{i} \lambda(1+\lambda)^{-\left(n-n_{i}\right)} e^{-\frac{p}{p_{0}} \lambda\left(\tau-\sigma_{i}\right)} \leq c \lambda_{i}\left(1+\lambda_{i}\right)^{-\left(n-n_{i}\right)} e^{-\frac{p}{p_{0}} \lambda_{i}\left(\tau-\sigma_{i}\right)} .
$$

From the condition (2.4) we know that equation $F(\lambda)=0$ has no root in $(0, \infty)$. Then from Theorem B we clearly see that every solution of Eq.(1.5) oscillates.

Now if we let $c_{i}=0, i=1,2, \ldots, n$, then we can obtain the following explicit sufficient and necessary conditions for Eq.(1.1) and Eq.(1.5).

Theorem 3. Let $c_{i}=0, i=1,2, \ldots, n$ and the conditions $H_{1}$ and $H_{2}$ be fulfilled. Then every solution of Eq.(1.1) oscillates if and only if

$$
-\lambda_{0}\left(1-\lambda_{0}\right)^{m} e^{-\frac{p}{p_{0}} \lambda_{0} \tau}+p_{0}>0,
$$

where $\lambda_{0}=\frac{\frac{p \tau}{p_{0}}+m+1-\sqrt{\left(\frac{p \tau}{p_{0}}+m+1\right)^{2}-\frac{4 p \tau}{p_{0}}}}{\frac{2 p \tau}{p_{0}}}$.

Proof. Sufficiency. The sufficiency follows directly from Theorem 1.

Necessity. Assume that the condition (2.6) does not hold, then

$$
-\lambda_{0}\left(1-\lambda_{0}\right)^{m} e^{-\frac{p}{p_{0}} \lambda_{0} \tau}+p_{0} \leq 0,
$$

where $\lambda_{0}=\frac{\frac{p \tau}{p_{0}}+m+1-\sqrt{\left(\frac{p \tau}{p_{0}}+m+1\right)^{2}-\frac{4 p \tau}{p_{0}}}}{\frac{2 p \tau}{p_{0}}}, 0<\lambda_{0}<1$. That is to say $F\left(\lambda_{0}\right) \leq 0$. Since $F(0)=p_{0}>0$, according to the continuity of $F(\lambda)$, there exists $\lambda_{1} \in$ 
$\left(0, \lambda_{0}\right)$ such that $F\left(\lambda_{1}\right)=0$ or $H\left(\frac{p_{0}}{p} \lambda_{1}\right)=0$. Therefore

$$
x(t)=e^{-\frac{p_{0}}{p} \lambda_{1} t}\left(1-\frac{p_{0}}{p} \lambda_{1}\right)^{-m}
$$

is a nonoscillatory solution of Eq.(1.1)

Remark 2. 1. If $p \rightarrow 0$, then $\lambda_{0} \rightarrow \frac{1}{m+1}$. In this case the condition (2.6) becomes

$$
p_{0} \geq \frac{m^{m}}{(m+1)^{m+1}} .
$$

This result is the same as [8, Theorem 7.2.1]. In fact, if $p=0$, then for each solution $x(t)$ of Eq.(1.1), there exists $n_{0} \in \mathbb{Z}$ such that $x(t) \equiv c_{k+1}$ for $t \in\left(t_{k}, t_{k+1}\right], k \geq n_{0}$. Here the sequence $\left\{c_{k}\right\}$ satisfies the difference equation

$$
c_{k+1}-c_{k}+p_{0} c_{k-m}=0 .
$$

The solution of (2.7) are oscillatory if and only if [8, Theorem 7.2.1].

$$
p_{0} \frac{(m+1)^{m+1}}{m^{m}}>1, m \in N .
$$

2. If $m=0$, then $\lambda_{1}=\frac{p_{0}}{p \tau}$. In this case we have the following differential equation without impulses:

$$
x^{\prime}(t)+p x(t-\tau)=0,
$$

and the condition (2.6) becomes

$$
-\frac{p_{0}}{p \tau} e^{-1}+p_{0}>0
$$

that is $p \tau e>1$. This result is the same as [8, Theorem 2.2.3].

Theorem 4. Let $c_{i}=0, i=1,2, \ldots, n$ and the conditions $H_{3}$ and $H_{4}$ be fulfilled. Then every solution of Eq.(1.5) oscillates if and only if

$$
\lambda_{0}\left(1+\lambda_{0}\right)^{-n} e^{-\frac{p}{p_{0}} \lambda_{1} \tau}+p_{0}<0,
$$

where $\lambda_{0}=\frac{-\left(\frac{p \tau}{p_{0}}+n-1\right)+\sqrt{\left(\frac{p \tau}{p_{0}}+n-1\right)^{2}+\frac{4 p \tau}{p_{0}}}}{\frac{2 p \tau}{p_{0}}}$.

Proof. The sufficiency follows directly from Theorem 2 and the proof of the necessity is similar to that of Theorem 3, so we omit it.

Remark 3. 1. If $p \rightarrow 0$, then $\lambda_{0} \rightarrow \frac{1}{n-1}$. The condition (2.7) becomes

$$
\frac{(n-1)^{n-1}}{n^{n}}+p_{0}<0, \quad n>1 .
$$

This result is the same as [8, Theorem 2.2.3].

2. If $n=0$, then $\lambda_{0}=\frac{p \tau}{p_{0}}$. In this case we have not impulses and the condition (2.7) becomes $F\left(\lambda_{0}\right)=\frac{p_{0}}{p \tau} e^{-1}+p_{0}<0$ or $1+p \tau e<0$. 


\section{References}

[1] R. P. Agarwal, M. Bohner, and W. T. Li, Nonoscillation and Oscillation: Theory for Functional Differential Equations, Marcel Dekker, New York, 2004.

[2] R. P. Agarwal, S. R. Grace, and D. O'Regan, Oscillation Theory for Difference and Functional Differential Equations, Kluwer Academic Publishers, Dordrecht, 2000.

[3] _ Oscillation Theory for Second Order Dynamic Equations, Taylor \& Francis, London, 2003.

[4] D. Bainov and D. P. Mishev, Oscillation Theory for Neutral Differential Equations with Delay, Adam Hilger, Bristol, 1991.

[5] D. Bainov and P. Simeonov, Oscillation Theory of Impulsive Differential Equations, International Publications, Orlando, 1998.

[6] L. H. Erbe, Q. K. Kong, and B. G. Zhang, Oscillation Theory for Functional-Differential Equations, Marcel Dekker, New York, 1995.

[7] K. Gopalsamy and B. G. Zhang, On delay differential equations with impulses, J. Math. Anal. Appl. 139 (1989), no. 1, 110-122.

[8] I. Győri and G. Ladas, Oscillation Theory of Delay Differential Equations, Oxford University Press, New York, 1991.

Jinfa Cheng

Department of Mathematics

Xiamen University

Xiamen 361005, P. R. ChinA

E-mail address: jfcheng@xmu.edu.cn

Yuming CHu

Department of Mathematics

Huzhou Teachers College

Huzhou 313000, P. R. China

E-mail address: chuyuming@hutc.zj.cn 